\section{Nanotechnology- based drug delivery in mucosal immune diseases: hype or hope?}

The use of nanotechnology (i.e., the manipulation and control of nanoscale objects ${ }^{1}$ ) is rapidly growing in medicine and has generated significant interest in mucosal immunology over the past few years. It has led to important innovations in vaccinology (e.g., ref. 2) and molecular imaging (e.g., ref. 3); in immunology, nanotechnology-based research is focused on the development of optimally designed therapeutic agents at mucosal surfaces. ${ }^{4} \mathrm{~A}$ desirable situation in mucosal drug delivery would be to have efficacious and autonomous drug-release devices that integrate with the human body without causing harm. The challenge is to maximize drug concentration for sufficient time at a particular mucosal location while minimizing systemic side effects. The use of nanomaterials creates endless possibilities for modifications in the properties of therapeutic agents, including solubility, surface tailorability, stability, multifunctionality, and immunogenicity. Thus, nanotechnologybased particles may hold great potential as novel drug carrier systems for targeted and controlled mucosal delivery and release.

By definition (http://nano.gov/ nanotech-101/what), nanomaterial is small (nanoscale: $1-100 \mathrm{~nm}$ ), but there is much debate regarding the suitability of this simple criterion. ${ }^{5}$ Critical parameters other than size may include shape, ratio of surface area to mass and related properties (e.g., surface charge), material composition, and functional phenomena. For pharmaceutical purposes, nanoparticles can range from 2 $\mathrm{nm}$ to $1 \mu \mathrm{m}$ (ref. 6). Thus far, major classes of nanomaterials used in drug delivery include liposomes, polymers, micelles, dendrimers, and metallic/ceramic nanoparticles. ${ }^{7}$
Ongoing preclinical research in mucosal immunology aims to engineer nanoparticles with biocompatible and biodegradable materials that carry anti-inflammatory substances, nucleic acids, or other molecules to diseased sites of specific mucosal tissues, such as the gastrointestinal tract, ${ }^{8}$ the lung airways, ${ }^{9}$ and the eye. ${ }^{10}$ Oral nanoparticle formulations may be designed to exhibit improved mucosa-adhesive properties, release the drug at a specific $\mathrm{pH}$ value, be resistant to luminal enzymes, or require bacterial cleavage for site-specific activation. For instance, Laroui et al. ${ }^{11}$ recently engineered polyactic acid nanoparticles loaded with anti-inflammatory tripeptide Lys-Pro-Val. The nanoparticles were encapsulated in a polymeric hydrogel (alginate/ chitosan) that was specifically degraded by digestive enzymes in the colon at pH 6.2. Using this improved oral nanoparticle-based drug delivery system, a 1,000-fold lower dose ${ }^{8}$ was sufficient to ameliorate mucosal inflammation in acute dextran sodium sulfate-induced (DSS) colitis in mice. In another study, ${ }^{12}$ the same group successfully used thioketal nanoparticles as an oral delivery vehicle for tumor necrosis factor- $\alpha$-small interfering RNA (siRNA) to block inflammation in acute DSS colitis. Thioketal derives from a polymer that degrades selectively in areas of high reactive oxygen species production, thus localizing the release of siRNA specifically to inflamed intestinal tissues. Although these reports show that oral administration of nanoparticle-based drugs ameliorates acute chemically induced colitis, it remains to be investigated whether it may also be efficient to prevent or treat chronic, spontaneous colitis. Others enriched human neutrophil-derived microparticles, which were used as scaffolds to construct a novel form of nanoparticles loaded with antiinflammatory aspirin-triggered resolving D1 or a lipoxin A4 analog. These "humanized" nanoparticles stimulated resolution of inflammation in acute models of peritonitis and temporomandibular arthritis in mice. ${ }^{13}$ It remains to be determined whether this innovative approach may also accelerate wound healing in inflamed mucosal tissues.
Despite this optimistic outlook on nanotechnology-based drug delivery, many hurdles must still be overcome. Naturally, the future nanoparticle-preparation process should be contaminant-free, standardized and reproducible, relatively inexpensive, and easy to scale up. To increase drug efficacy and ensure patient compliance, the nanocarrier formulation must be safe, simple to administer, and, most important, nontoxic. Very little is known about the potential effects of nanoparticles and individual components on the human immune system (not to mention with long-term administration). Protective mucus usually traps and removes foreign particles from the mucosal surface. Biodegradable polymeric particles of larger size (200 nm) have been shown to be capable of rapidly penetrating healthy (e.g., cervicovaginal ${ }^{14}$ ) or diseased (e.g., chronic rhinosinusitis ${ }^{15}$ ) human mucus barriers. During this process, however, nanoparticles can alter the microstructure of the mucus barrier, ${ }^{16}$ but the functional impact of this observation remains to be examined in vivo (e.g., do nanoparticle-induced "holes" disrupt the mucus barrier, allowing bacterial translocation?). Once the mucus is crossed, nanoparticles should not cause toxicity to the epithelial cells or immune hyper-/ hyposensitivity in the underlying lamina propria.

Nanoparticle-bound drugs comprise multiplexed formulations (e.g., drug, targeting moiety, scaffold material) with a wide variety of characteristics. Distinct nanoparticles can exploit different mechanisms to gain entry into a cell. ${ }^{17}$ Rather than passing directly through a cell wall, they may be actively endo- or phagocytosed. Nanoparticles' many physicochemical and biological properties (not only size, shape, and material) could modulate the individual cell immune response, ${ }^{18}$ which at this point largely represents a "black box" in mucosal tissues. Comprehensive data on immunomodulatory profiles of biocompatible organic nanoparticles in mucosal immune cells are lacking. Establishing standardized and meaningful in vitro immunotoxicological assays for nanomaterials is challenging. ${ }^{19}$ 
Currently, any foreign substance at mucosal surfaces should be considered with caution. Some metal or inorganic material nanoparticles regarded as biologically inert may nevertheless be recognized as "non-self" by the human innate immune system. An example is titanium dioxide, which has recently been shown to activate the inflammasome, causing pulmonary inflammation. ${ }^{20}$

In conclusion, recent advances have highlighted the great promise of nanoparticlebased drug delivery for the treatment of mucosal immune diseases. But it is clear that nanotechnology-based drug therapy strategies at mucosal surfaces are not likely to reach the clinic in the very near future. The emerging field of "nanoimmunology" is still in its infancy, opening a multitude of exciting research opportunities for mucosal immunologists as well as those in other disciplines. A comprehensive understanding of the complex mechanistics and differential subcellular effects of nanoparticles on the mucosal immune system (healthy vs. diseased) is now needed. Study findings may help to realize targeted nanodelivery of a drug to a particular mucosal cell subset. In this context, future investigations of nanotechnology-based therapy at mucosal sites should also consider completely new approaches, such as genetic conversion devices, ${ }^{21}$ which could possibly reprogram aberrant cell signaling in mucosal disorders.

\section{Elke Cario, Associate Editor}

(C) 2012 Society for Mucosal Immunology

\section{REFERENCES}

1. MCNeil, S.E. Unique benefits of nanotechnology to drug delivery and diagnostics. Methods Mol. Biol. 697, 3-8 (2011).

2. Kasturi, S.P. et al. Programming the magnitude and persistence of antibody responses with innate immunity. Nature 470, 543-547 (2011).

3. Hikage, M. et al. Nano-imaging of the lymph network structure with quantum dots. Nanotechnology 21, 185103 (2010).

4. de la Fuente, M. et al. Nanoparticles as protein and gene carriers to mucosal surfaces. Nanomedicine (Lond) 3, 845-857 (2008).

5. Maynard, A.D. Don't define nanomaterials. Nature 475, 31 (2011).

6. Bawarski, W.E. et al. Emerging nanopharmaceuticals. Nanomedicine 4, 273282 (2008).

7. Zhang, L. et al. Nanoparticles in medicine: therapeutic applications and developments. Clin. Pharmacol. Ther. 83, 761-769 (2008).

8. Laroui, H. et al. Nanomedicine in Gl. Am. J. Physiol. Gastrointest. Liver Physiol. 300, G371G383 (2011).

9. Roy, I. \& Vij, N. Nanodelivery in airway diseases: challenges and therapeutic applications. Nanomedicine 6, 237-244 (2010).

10. du Toit, L.C. et al. Ocular drug delivery-a look towards nanobioadhesives. Expert Opin. Drug Deliv. 8, 71-94 (2011).

11. Laroui, H. et al. Drug-loaded nanoparticles targeted to the colon with polysaccharide hydrogel reduce colitis in a mouse model. Gastroenterology 138, 843-853.e1-2 (2010).

12. Wilson, D.S. et al. Orally delivered thioketal nanoparticles loaded with TNF-alpha-siRNA target inflammation and inhibit gene expression in the intestines. Nat. Mater. 9, 923-928 (2011).

13. Norling, L.V. et al. Cutting edge: humanized nano-proresolving medicines mimic inflammation-resolution and enhance wound healing. J. Immunol. 186, 5543-5547 (2011).

14. Tang, B.C. et al. Biodegradable polymer nanoparticles that rapidly penetrate the human mucus barrier. Proc. Natl. Acad. Sci. USA 106, 19268-19273 (2009).

15. Lai, S.K. et al. Drug carrier nanoparticles that penetrate human chronic rhinosinusitis mucus. Biomaterials 32, 6285-6290 (2011).

16. Wang, Y.Y. et al. Mucoadhesive nanoparticles may disrupt the protective human mucus barrier by altering its microstructure. PLoS One 6:e21547 (2011).

17. Hess, H. \& Tseng, Y. Active intracellular transport of nanoparticles: opportunity or threat? ACS Nano 1, 390-392 (2007).

18. Dwivedi, P.D. et al. Impact of nanoparticles on the immune system. J. Biomed. Nanotechnol. 7, 193-194 (2011).

19. Oostingh, G.J. et al. Problems and challenges in the development and validation of human cell-based assays to determine nanoparticleinduced immunomodulatory effects. Part. Fibre Toxicol. 8, 8 (2011).

20. Yazdi, A.S. et al. Nanoparticles activate the NLR pyrin domain containing 3 (NIrp3) inflammasome and cause pulmonary inflammation through release of IL-1alpha and IL-1beta. Proc. Natl. Acad. Sci. USA 107, 19449-19454 (2010).

21. Ye, H. et al. A synthetic optogenetic transcription device enhances blood-glucose homeostasis in mice. Science 332, 1565-1568 (2011). 